International Mathematical Forum, Vol. 9, 2014, no. 19, 947 - 952

HIKARI Ltd, www.m-hikari.com

http://dx.doi.org/10.12988/imf.2014.4114

\title{
On Necessary Conditions for Scalars
}

\author{
D. O. Adicka \\ Department of Mathematics and Computer Science \\ University of Kabianga, Box 2030 Kericho, Kenya \\ W. Mukuna \\ Department of Mathematics and Computer Science \\ University of Kabianga, Box 2030 Kericho, Kenya \\ P. O. Oleche \\ Department of Mathematics \\ Maseno University, Box 333 Maseno, Kenya
}

Copyright (c) 2014 D. O. Adicka, W. Mukuna and P. O. Oleche. This is an open access article distributed under the Creative Commons Attribution License, which permits unrestricted use, distribution, and reproduction in any medium, provided the original work is properly cited.

\begin{abstract}
In this paper, we give a characterization of scalar operators. In particular we show that a densely defined closed linear operator $H$ acting on a reflexive Banach space $X$ is scalar if it is of $(0,1)$ type $\mathbb{R}$ and $\|f(H)\| \leq\|f\|_{\infty}$ for $f$ in the algebra of smooth functions $\mathcal{U}$.
\end{abstract}

Keywords: Reflexive Banach space, A densely defined operator

\section{Introduction}

Suppose $H$ is a closed densely defined operator on a Banach space $X$, whose spectrum is contained in $\mathbb{R}$ and there exist a $C>0$ such that

$$
\left\|(z-H)^{-1}\right\| \leq C \frac{<z>^{\alpha}}{\left|\mathcal{I}_{z}\right|^{\alpha+1}}
$$


for all $z \in i \mathbb{R}$ and some $(\alpha, \alpha+1) \geq 0$ then $H$ is of $(\alpha, \alpha+1)$ type $\mathbb{R}[1]$.

Here, $\langle z\rangle:=\sqrt{1+|z|^{2}}$ and $\mathcal{I} z$ denotes the imaginary part of $z$.

A special case is a Hermitian operator on a Hilbert space. Scalar operators with real spectrum is called a pseudo-hermitian operator. In Hilbert space, abounded linear operator $S$ is a pseudo-hermitian if and only if the group $\left\|e^{i t S}\right\| \leq M<\infty$ for all $t \in \mathbb{R}[8]$.

If $X$ is a reflexive Banach space then an operator $T \in B(X)$ is scalar spectral if it admits an integral representation with respect to countably additive projection valued measure or equivalently if it admits a $C(\sigma(T))$ functional calculus [6]. In particular, if $T$ acts on a Hilbert space $\mathcal{H}$, then $T$ admits $C(\mathbb{R})$ functional calculus if it is Hermitian. Generally, an operator acting on a reflexive Banach space is scalar if and only if it has a $C_{o}(\mathbb{R})$ functional calculus [5]. According to [10], If $T$ is an operator with $\sigma(T) \subset \mathbb{R}$ and acting on a reflexive Banach space $X$, then $T$ is scalar if and only if $i H$ generates a uniformly bounded strongly continuous group. In [7], a functional calculus is given for a closed densely defined linear operators on a Banach space with $\sigma(H) \subseteq \mathbb{R}$ satisfying the resolvent estimate and for functions from weighted sobolev spaces. Here the calculus used is based on almost analytic extension to $\mathbb{C}$ of infinitely differentiable functions defined on $\mathbb{R}$ and the Helffer-Sjostrand formula [9]. Such calculus defines an algebra homomorphism. We now consider an intermediate topology $C_{c}^{\infty}(\mathbb{R}) \subset \mathcal{U} \subseteq C(\mathbb{R})$ such that $(\alpha, \alpha+1)$ type $\mathbb{R}$ operators admits $\mathcal{U}$ functional calculus. Here $C_{c}^{\infty}(\mathbb{R})$ is the space of smooth functions of compact support. For detailed information see [2].

For any $f \in \mathcal{U}$ the norm is defined as;

$$
\|f\|_{n}:=\sum_{r=0}^{n} \int\left|f^{(r)}(x)\right|<x>^{r-1} d x
$$

where

$$
\left|f^{(r)}(x)\right|:=\left|\frac{d^{r}}{d x^{r}} f(x)\right| \leq c_{r}<x>^{\beta-r}
$$

for all $x, \beta \in \mathbb{R}$ and $c_{r}>0$.

It is shown in [2] that $\mathcal{U}$ is an algebra under pointwise multiplication.

The definition of $f(H)$ for $f \in \mathcal{U}$ originates from the version of Helffer and Sjöstrand [9] integral formula. Using this and the abstract result from [3], we show that a densely defined closed linear operator $H$ acting on a reflexive Banach space $X$ is scalar if it is of $(0,1)$ type $\mathbb{R}$ and $\|f(H)\| \leq\|f\|_{\infty}$ 


\section{The $\mathcal{U}$ functional Calculus}

The materials in this section has been taken from [3] and [4].

For any $f \in \mathcal{U}$ and $n \geq 0$ an almost analytic extension of $f$ to $\mathbb{C}$ is defined;

$$
\widetilde{f}(x+i y):=\sum_{r=0}^{n} \frac{f^{(r)}(x)(i y)^{r}}{r !} \tau\left(\frac{y}{<x>}\right)
$$

where $\tau$ is a $\mathcal{C}_{c}^{\infty}(\mathbb{R})$ function such that $\tau(s)=1$ if $|s| \leq 1$ and $\tau(s) \geq 2$.

It follows that for $f \in \mathcal{U},\left|\frac{\partial}{\partial \bar{z}} \tilde{f}(x, y)\right|=\mathcal{O}\left(|y|^{n}\right)$ as $|y| \rightarrow 0$ for a fixed $x$. Moreover we can find $c^{\prime} \in \mathbb{R}$ such that

$$
\left|\frac{\partial}{\partial \bar{z}} \widetilde{f}(x, y)\right| \leq c^{\prime}\left(|y|^{n}\right)
$$

as $z \rightarrow x \in \mathbb{R}$. If $\kappa$ is a map such that $\kappa: \mathcal{U} \rightarrow B(X)$ then

$$
f \rightarrow f(H):=-\frac{1}{\pi} \int_{\mathbb{C}} \frac{\partial \tilde{f}}{\partial \bar{z}}(z-H)^{-1} d x d y
$$

and it is proved in [3], that for $n>\alpha \geq 0$

- $f(H)$ is norm convergent with $\|f(H)\| \leq C_{\alpha}\|f\|_{n+1}$ for some $C_{\alpha}>0$ and doesn't depend on $\tau$;

- the mapping extends to a bounded algebra homomorphism;

- if $f \in \mathcal{U}$ and $f=0$ on a neighbourhood of $\sigma(H)$ then $f(H)=0$;

- if $z \in i \mathbb{R}$ then $\frac{1}{z-} \in \mathcal{U}$ and $f\left(\frac{1}{z-}\right)=(z-H)^{-1}$

For an operator $H$ of $(\alpha, \alpha+1)$-type $\mathbb{R}$, we associate each element $f \in \mathcal{U}$ with an operator $f(H) \in B(X)$ given by $(6)$

In order to state our results, we need the following theorems and corollaries;

Theorem 2.1 Let $H$ be a bounded operator with $\sigma(H) \subseteq \mathbb{R}$, and $\left\|e^{i H t}\right\| \leq C(1+|t|)^{\alpha}$ where $\alpha$ is a non negative integer. Then $H$ is of $(\alpha, \alpha+1)$ type $\mathbb{R}$

Proof. see[1]

corollary 2.2 If $\alpha=0$ then $H$ is of $(0,1)$ type $\mathbb{R}$ and $\left\|e^{i H t}\right\| \leq C<\infty$. In particular $H$ is a pseudo hermitian operator, and so it is a scalar operator.

Theorem 2.3 $\mathrm{H}$ is a generator of a $C_{o}$-contraction semi-group if and only if $H$ is closed, densely defined and for each $\lambda>0, \lambda \in \rho(H)$ and $\left\|(\lambda-H)^{-1}\right\| \leq \lambda^{-1}$ 
Proof. see[1]

corollary 2.4 If $i H$ is a generator of a group of isometries $\{T(t)\}$ then for all $\lambda \in i \mathbb{R}$ with real $\lambda \neq 0, \lambda \in \rho(i H)$ and

$$
(\lambda-i H)^{-1}= \begin{cases}\int_{0}^{\infty} T(t) e^{-\lambda t} d t, & \text { if } R \lambda>0 \\ -\int_{0}^{\infty} T(t) e^{-\lambda t} d t, & \text { if } R \lambda<0\end{cases}
$$

Theorem 2.5 If $H$ is a Hermitian operator on a Hilbert space $\mathcal{H}$, then $H$ is of $(0,1)$ type $\mathbb{R}$

The proof of this theorem follows from the fact that since $H$ is a Hermitian operator then obviously its spectrum is in $\mathbb{R}$ and so the resolvent set of $H$; $\rho(H):=\{z \in i \mathbb{R}: z-H: \mathcal{D}(H) \rightarrow X$ is bijective and

$\left.(z-H)^{-1} \in B(X)\right\}$. In particular $\left\|(z-H)^{-1}\right\| \leq C|\mathcal{I} z|^{-1}$ by (1); thus for all $z \in \rho(H), R(z, H):=(z-H)^{-1}$ is a normal operator.

Theorem 2.6 If $H$ is of $(\alpha, \alpha+1)$-type $\mathbb{R}$ for some $\alpha>0$, then $H$ admits $C_{o}^{\infty}(\mathbb{R})$ functional calculus.

Proof. see [1]

Theorem 2.7 If $f \in \mathcal{U}$ and $H$ is Hermitian on a Hilbert space $\mathcal{H}$, then

$$
\|f(H)\| \leq\|f\|_{\infty}
$$

Proof. See[3]

\section{$3 \quad$ Main Results}

Theorem $2.8 H$ is of $(0,1)$-type $\mathbb{R}$ with the constant $C=1$ if and only if $i H$ is a generator of a one parameter group of isometries on $X$.

Proof.

Suppose $H$ is of $(0,1)$-type $\mathbb{R}$ with $C=1$, then from corollary (2.2), $H$ is a Pseudo-Hermitian operator, and hence a scalar operator. It follows from (1) and (Theorem 2.1) that $H$ is a generator of a one parameter group of isometries and so $i H$ also generates a group of isometries. Since $i H$ generates a group of isometries it follows that $i H$ is densely defined. Also from corollary(2.4); we have that for $\lambda>0 ;(\lambda-i H)^{-1} f$ is the Laplace transform of $T(t)=e^{i t H} f$ given for $f$ in the domain of $i H$. It follows from theorem $(2.1)$ that $T(t)$ is a bounded operator. Conversely, suppose $i H$ densely defined and theorem (2.1) holds, then $T(t)$ is a semigroup. From the uniform boundedness, $\left(T(t)_{t \geq 0}\right)$ is uniformly bounded on compact intervals. From corollary (2.4) and density of $D(i H)$, imply that $(T(t))$ is strongly continuous. Hence $i H$ is a generator of 
one parameter group $T$.

Theorem 2.9 A densely defined linear operator $H$ acting on a reflexive Banach space $X$, is scalar if it is of $(0,1)$-type $\mathbb{R}$ and $\|f(H)\| \leq\|f\|_{\infty}$ for each $f \in \mathcal{U}$

Proof. Let $H$ be an operator acting on Hilbert space $\mathcal{H}$ and $\sigma(H) \subseteq \mathbb{R}$, then $H$ is A Pseudo Hermitian Operator. By theorem $(2.5)$ it is of $(0,1)$-type $\mathbb{R}$ and by theorem (2.6) it is a scalar operator. Also $i H$ generates a one parameter group by theorem 2.8, hence $H$ admits a functional calculus given by (6). Since (6) is continuous by (1) and (5), the resolvent set is bounded. From theorem (2.7) we see that (6) converges absolutely. Since $H$ is Hermitian, it follows by Riesz representation theorem that for $f \in \mathcal{U}$ there exist a complex Borel measure $\mu$ on $\sigma(H)$ such that

$$
f(H)=\int_{\sigma(H)} f(z) \mu d z
$$

and this completes the proof.

Acknowledgements. The first author (D. O. Adicka) would like to thank University of Kabianga (Kenya) for assistantship during the period of research.

\section{References}

[1] P O. Oleche, N. O Ongati and J. O Agure. Operators with slowly growing resolvents towards the spectrum. International Journal of Pure and Applied Mathematics,51(3):245-357, March 2009.

[2] P O. Oleche, N. O Ongati and J. O Agure. The algebra of smooth functions of rapid descent. International Journal of Pure and Applied Mathematics,52(2):163-176, 2009.

[3] P O. Oleche, J. O Agure. A functional Calculus for $(\alpha, \alpha+1)$ - type $\mathbb{R}$ Operators Int. Journal of Math. Analysis,vol.4,2010,no,737-760

[4] A.Batkai and E.Fasanga The spectral mapping theorem for Davis' functional calculus AMS (2000),1-7.

[5] H.R. Dowson. Spectral Theory of Linear Operators. Academic Press, New York, 1978.

[6] I. Doust and G.Lancien The spectral type of sums of operators on nonHilbertian Banach lattices.J.Aust. Math Soc.84(2008),193-198. 
[7] E.B. Davies, The functional calculus. J. London Math. Soc. 52(1995),166176.

[8] S. Kantorovitz On the characterization of spectral operators.Trans.Amer.Math.Soc.111(1964),152-181.

[9] Helffer B. and Sjöstrand, J. Euation de Schrodinger Operator avec magnetique et equation de Harper,345 Of Lecture notes in Physics,SprinerVerlag,Berline, 1989.

[10] S.Kantorovitz. Spectral theory of Banach space Operators, volume 1012 of Lecture notes in Mathematics. Springer-Verlag, Berlin, 1989.

\section{Received: January 15, 2014}

